\title{
Effects of different EMS solution concentration and time treatment on morphological traits of Cyperus esculentus $\mathrm{L}$
}

\author{
Yan $\mathrm{Ye}^{1}$, Xueying $\mathrm{Li}^{1}$, Hailin Qian ${ }^{1}$, Songnan Yang ${ }^{1}$, Xiao Han ${ }^{1}$, and Jun Zhang, * \\ ${ }^{1}$ Jilin Agricultural University, Changchun City, People's Republic of China
}

\begin{abstract}
Absrtact. In order to study the effect of concentration and time of EMS solution on the emergence and performance of agricultural characters of Cyperus esculentus L., Jinong oil 6 was used as the experimental material, and different concentration $(0.5 \%, 0.7 \%, 0.9 \%)$ and different treatment time (6h, 12h, 24h) were set to treat the seeds of Cyperus esculentus L.. The results showed that the seedling emergence rate, 100 seed weight and seed volume had different performance under different solution concentration and time treatment, and there was strong correlation between them. It was preliminarily considered that the higher solution concentration and time treatment had greater influence on the performance of agricultural characteristics of Cyperus esculentus L., and the combination of $0.9 \%$ solution concentration and $6 \mathrm{~h}$ time was the best condition for EMS induction, tiller number, plant height, yield and other agronomic characters are the most best.
\end{abstract}

\section{Foreword}

\subsection{Economic value of Cyperus esculentus $\mathrm{L}$.}

Cyperus esculentus L., also known as Cyperus and Cyperus perennial plant, is a genus of Cyperus in the Family Cyperaceae, native to Africa, it is resistant to waterlogging, drought, poor quality ${ }^{[1]}$, high oil content, showing good adaptability, strong resistance to adversity, outstanding yield, excellent oil quality and so on ${ }^{[2]}$. It has high economic value of gathering forage, grain and oil as a whole, be crowned with"King of Oil Crop"laudatory name ${ }^{[3]}$.

\subsection{The research progress of Cyperus esculentus L.at home and abroad}

A great deal of research has been done on the morphological characteristics, developmental characteristics, growth habits, components and planting conditions of Cyperus esculentus L. at home and abroad, and some achievements have been made, the tissue culture, regeneration and genetic transformation system of Cyperus esculentus L. have not been established, which limits the genetic improvement of Cyperus esculentus L. by genetic

* Corresponding author: zhangjun@jlau.edu.cn 
engineering $^{[4]}$.

\subsection{Mutation breeding techniques}

Mutation breeding includes physical mutation and chemical mutation, which is a method of using chemical or physical factors to cause crop variation and breeding new varieties by selection according to people's breeding goal. Chemical Mutagenesis has been widely used in induced mutation breeding for its high mutation rate, high mutation frequency and high specificity ${ }^{[5]}$.

Ethyl methanesulfonate (EMS) is a good chemical attractant, which has been widely used in various crops to obtain abundant variants. Wang Yun ${ }^{[6]}$ used EMS to study the change trend of agronomic characters and quality characters of M1 and M2, which laid a foundation for constructing mutant library and breeding new peanut varieties with high yield and good quality The EMS Mutagenesis of FengWuyi ${ }^{[7]}$ and Dingyanqing ${ }^{[8]}$ provided $^{2}$ an important germplasm source for wheat breeding, which provided a new way to search for the key genes of protein and lipid in soybean seed. EMS mutagenesis is most commonly applied to seeds, which can be treated in large quantities without complicated process and is characterized by short time-consuming and simple tissue culture ${ }^{[9]}$.

In this study, Jinong Oil 6 was used as the test material, and the concentration and time of EMS solution were determined, the effects of each treatment combination on seedling rate, plant height, 100-seed weight, seed volume and yield were observed and compared, it provides a theoretical reference for expanding the high quality germplasm resources of Cyperus esculentus L. and establishing the variant resources of Cyperus esculentus L. and selecting good new varieties.

\section{Materials and methods}

\subsection{Test material}

The seeds used in the experiment were collected by the cash crop research team of Jilin Agricultural University for the preservation of Jilin province's large-scale planting material "Jinong Oil 6" .

The MUTAGEN, produced by Emssigma-alorich, is a colorless liquid.

\subsection{EMS solution preparation[10]}

(1) Using $22.82 \mathrm{G} \mathrm{K} 2 \mathrm{HPO} 43 \mathrm{H} 2 \mathrm{O}$ and $13.6 \mathrm{G} \mathrm{KH} 2 \mathrm{PO} 4$ and distilled water to prepare 200 ML $1 \mathrm{~mol} / \mathrm{L}$ Dipotassium phosphate solution and $100 \mathrm{ML} 1 \mathrm{~mol} / \mathrm{L}$ Monopotassium phosphate solution respectively.

(2) Phosphoric acid buffer was prepared from 70ML K2HPO4 solution and 30ML $\mathrm{KH} 2 \mathrm{PO} 4$ in $100 \mathrm{ml}$ volumetric flask, and then diluted 10 times in 1L volumetric flask.

(3) The phosphoric acid buffer and EMS were prepared into a mixture of $0.5 \%, 0.7 \%$ and $0.9 \%$ respectively, which was repeated three times.

\subsection{Seed volume measurement}

Put 100 seeds in the water and divide by the volume of water. 


\subsection{Experimental design}

Two treatment factors were set up: 3 EMS solution concentrations $0.5 \%, 0.7 \%, 0.9 \%$, respectively numbered $1,2,3 ; 3$ Treatment Times $(6 \mathrm{~h}, 12 \mathrm{~h}, 24 \mathrm{~h})$, respectively numbered A, B, C; 1 Control Group distilled water soaked for 24 hours, respectively, numbered CK, there are 10 combinations.

The experiment was carried out in the appraisal station $\left(125.4^{\circ} \mathrm{e}, 43.8^{\circ} \mathrm{n}\right)$ and laboratory of Jilin Agricultural University. Ten 100 full, intact, non-damaged, insect-free, uniform-sized seeds were soaked in distilled water at $35{ }^{\circ} \mathrm{C}$ for 3 days. One Part was control group without any treatment, the other 9 parts were put into the corresponding concentration of EMS solution and soaked for the corresponding time. All seeds are soaked and rinsed with water for 2 hours.

On May 26, 2019, the fields were seeded in random groups to keep the same water and fertilizer conditions, and each treatment was repeated 3 times. The emergence rate, maximum tiller number and plant height of Cyperus esculentus L.induced by EMS were investigated.

October 9, 2019 harvest materials, wash to remove impurities and dry, indoor test species and record under each treatment of the performance and yield.

\subsection{Data processing and statistical analysis}

Excel 2010 and SPSS were used for data statistical analysis and mapping. The data were analyzed by two-way Anova and Pirsson correlation analysis $(\mathrm{p}<0.05)$.

\section{Results and analysis}

\subsection{Effects of EMS on the emergence rate, 100-seed weight and seed volume of Cyperus esculentus $\mathrm{L}$.}

As can be seen from Table 1, the emergence rate, 100 -seed weight and seed volume of Cyperus esculentus L.were significantly different under different treatment time, but only 100 -seed weight was significantly different under different solution concentration, the difference of emergence rate and seed volume was not significant. The results showed that the emergence rate, 100-seed weight and seed volume of Cyperus esculentus L.were different under different conditions. Under the treatment time and concentration, there was no significant difference in the emergence rate and seed volume, but significant difference in 100-seed weight, which was different from that of the treatment time and concentration alone, this may be due to the interaction of two experimental factors, treatment time and treatment concentration. It further indicates that there may be interaction between treatment time and treatment concentration of EMS solution.

In order to study the correlation among emergence rate, 100-seed weight and seed volume of Cyperus esculentus L. after EMS treatment, Pirsson correlation analysis was carried out with SPSS software (table 2). It can be seen from table 2 that the emergence rate, 100-seed weight and seed volume of Cyperus esculentus L.show a strong positive correlation. This will facilitate the selection of breeding in the future, in the breeding selection in the future, we can give priority to the selection of large seed individuals to reproduce, to achieve high seedling rate and 100-seed weight are better agronomic traits, in order to get a higher yield. 
Table 1. Significance of the number of seedlings, 100 grain weight and seed volume processed by SPSS software.

\begin{tabular}{|l|c|c|c|}
\hline Significance ( Sig. ) & Emergence rate & Hundred Grain Weight & Volume \\
\hline Processing Time & 0.001 & 0.000 & 0.000 \\
\hline Treatment concentration & 0.500 & 0.006 & 0.111 \\
\hline $\begin{array}{l}\text { Treatment Time } \\
\text { Treatment Concentration }\end{array}$ & 0.188 & 0.004 & 0.024 \\
\hline
\end{tabular}

Significance level 0.05

Table 2. Pearson correlation of the number of seedlings, 100 grain weight and seed volume processed by SPSS software.

\begin{tabular}{|c|c|c|c|c|}
\hline \multicolumn{2}{|c|}{ Indicators } & Emergence rate & Hundred Grain Weight & Volume \\
\hline \multirow{2}{*}{$\begin{array}{c}\text { Emergence } \\
\text { rate }\end{array}$} & Pirsson correlation & 1 & $0.618^{* *}$ & $0.715^{* *}$ \\
\cline { 2 - 5 } & Sig.(two tails) & & 0.001 & 0.000 \\
\hline \multirow{2}{*}{$\begin{array}{c}\text { Hundred } \\
\text { Grain Weight }\end{array}$} & Pirsson correlation & $0.618^{* *}$ & 1 & $0.632^{* *}$ \\
\cline { 2 - 5 } & Sig.(two tails) & 0.001 & & 0.000 \\
\hline \multirow{2}{*}{ Volume } & Pirsson correlation & $0.715^{* *}$ & $0.632^{* *}$ & 1 \\
\cline { 2 - 5 } & Sig.(two tails) & 0.000 & 0.000 & \\
\hline
\end{tabular}

**. On a scale of 0.01 (double-tailed), the correlation was significant.

\subsection{The effects of EMS on Tiller number and plant height of Cyperus esculentus L. were studied}

As a whole, the tiller number of Cyperus esculentus L.showed different fluctuation under the treatment of different EMS concentration and time, with the increase of EMS concentration and treatment time, the number of tillers of Cyperus esculentus L.decreased gradually, the number of tillers of CK was the highest in control group, followed by those of A3 and B2 treatments, and the lowest in C3 treatments. See figure 1-a for different EMS concentration treatments, under the same solution concentration, the tiller number of different time also has the difference: under the treatment of $0.5 \%, 0.7 \%, 0.9 \%$ solution concentration, the treatment time from high to low is $6 \mathrm{H}>24 \mathrm{~h}>12 \mathrm{~h} ; 12 \mathrm{~h}>6 \mathrm{~h}>24 \mathrm{~h} ; 6 \mathrm{~h}>$ $24 \mathrm{~h}>12 \mathrm{~h}$. The Tiller numbers of Cyperus esculentus L.treated with $0.5 \%$ and $0.7 \%$ solution concentration were similar, but the tiller numbers of Cyperus esculentus L. treated with $0.9 \%$ solution concentration were significantly different. This indicated that there was no linear relationship between tiller number and treatment time of EMS solution under the same treatment concentration. Similarly, it can be concluded in Fig. 1-b that there is no linear relationship between EMS solution concentration and tiller number under the same treatment.

The yield of Cyperus esculentus L.was related to whether the stem and leaf were cut or not. The yield of Cyperus esculentus L.was increased by 30\% compared with that of Cyperus esculentus L.without treatment. In order to maximize the yield of Cyperus esculentus L., the yield of Cyperus esculentus L.should be increased by cutting before harvest. From Fig. 2, it can be seen that the plant height of the control group was the highest from the seedling stage to the time before cutting. The order of plant height from large to small was $\mathrm{CK}>\mathrm{A} 1>\mathrm{A} 2>\mathrm{B} 1>\mathrm{A} 3>\mathrm{B} 3>\mathrm{C} 2>\mathrm{B} 2>\mathrm{C} 1>\mathrm{C} 3$ on August 20 before cutting, this shows that there is a certain interaction between the treatment concentration and treatment time. As a whole, the plant height of a series treatment group (A1, A2, A3) was higher than that of $\mathrm{c}$ series treatment group $(\mathrm{C} 1, \mathrm{C} 2, \mathrm{C} 3)$, this showed that there was a 
certain linear relationship between treatment time and plant height, but there was not a certain linear relationship between treatment concentration and plant height, it is suggested that the mutagenic treatment time level may have a greater effect on the plant height of Cyperus esculentus L..

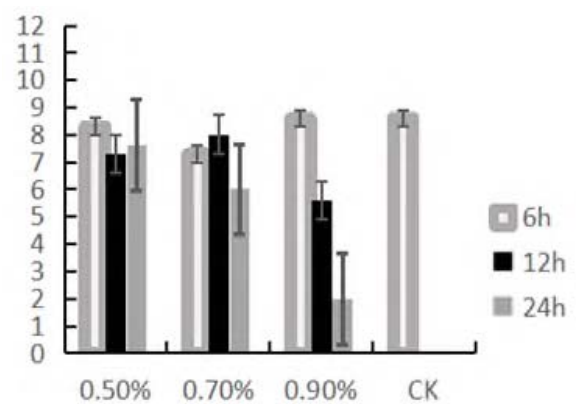

a

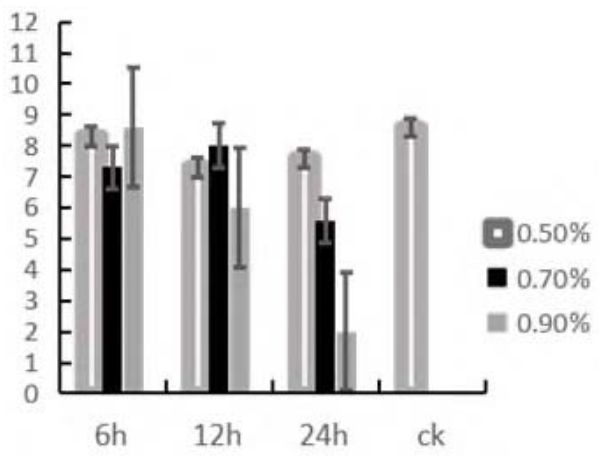

b

Fig.1. Tillering number of different concentration EMS solution treatment and different time treatment of Cyperus esculentus L.combination.

Note: figure a shows the effect of EMS concentration on Tiller number of Cyperus esculentus L.and figure B shows the effect of Em treatment time on tiller number of Cyperus esculentus L.

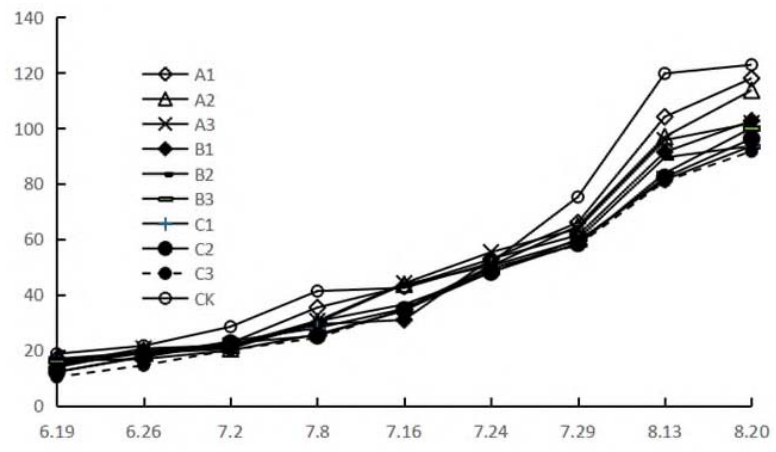

Fig. 2. Effect of different EMS treatments on the plant height of Cyperus esculentus L.

\subsection{The effects of EMS on the yield of Cyperus esculentus L. were studied}

The yield of Cyperus esculentus L. was affected by Tiller number, 100-seed weight and plant height. Using the Duncan test of SPSS software to mark the significance $(\mathrm{p}<0.05$; different letters of the mark showed significant difference) as shown in Table 3: The Control Group had the highest yield, the A3 treatment group was next, and the C3 treatment group had the lowest yield, it can be concluded that the highest treatment concentration gradient and the highest treatment time gradient have the greatest influence on the oil bean. Except the A3 treatment group, there were significant differences between the other 8 treatment groups and the control group. It can be seen that the yield of A3 treatment group was less influenced by EMS. The emergence rate was closely related to the yield, and the 
yield was positively related to the emergence rate. The higher the emergence rate, the higher the yield.

Table 3. The yield of different concentration EMS solution treatment and different time treatment of Cyperus esculentus L. combination by SPSS software.

\begin{tabular}{|c|c|c|}
\hline Deal with & Emergence rate & Yield per kg / Kg \\
\hline CK & $76.9 \pm 7.7 \%$ & $383.0 \pm 8.3^{\mathrm{a}}$ \\
\hline A3 & $74.4 \pm 11.8 \%$ & $371.7 \pm 20.8^{\mathrm{a}}$ \\
\hline A1 & $71.8 \pm 4.4 \%$ & $337.3 \pm 7.7^{\mathrm{b}}$ \\
\hline B1 & $66.7 \pm 8.9 \%$ & $317.9 \pm 15.1^{\mathrm{bc}}$ \\
\hline A2 & $61.5 \pm 0.0 \%$ & $308.6 \pm 6.4^{\mathrm{c}}$ \\
\hline B2 & $59.0 \pm 11.8 \%$ & $262.3 \pm 4.5^{\mathrm{d}}$ \\
\hline C1 & $48.7 \pm 4.4 \%$ & $230.2 \pm 17.9^{\mathrm{e}}$ \\
\hline C2 & $51.3 \pm 4.4 \%$ & $223.2 \pm 10.0^{\text {ef }}$ \\
\hline B3 & $48.7 \pm 11.8 \%$ & $203.1 \pm 2.9^{\mathrm{f}}$ \\
\hline C3 & $30.8 \pm 20.4 \%$ & $119.7 \pm 9.7^{\mathrm{g}}$ \\
\hline
\end{tabular}

\section{Discuss and conclsion}

\subsection{Discussion}

Cyperus esculentus L. is a new oil crop which is widely used in China. It has strong adaptability and high yield. It can be pressed oil and used as raw material to produce biodiesel. High Quality of germplasm resources is an important prerequisite for all kinds of research. In Crop Breeding, it is an important step to construct a genotype-rich mutant population and select good individuals from it. EMS is an excellent Mutagen because of its low breeding cost, high mutation frequency and simple operation process. The EMS Mutant Library of Arabidopsis Thaliana ${ }^{[12]}$, wheat ${ }^{[13,14]}$ and rice ${ }^{[15]}$ has been successfully constructed, it also plays an important role in breeding genes, but the research of EMS mutagenesis in Cyperus esculentus L. is still in its infancy. EMS mutagenesis may activate genes that inhibit seed germination or change the expression of genes that regulate seed germination ${ }^{[16]}$. EMS mutagenesis had significant effects on seed germination rate, seedling emergence rate and morphological characteristics ${ }^{[17]}$. Therefore, the emergence rate, tiller number, 100-seed weight, seed volume and plant height of the treatment group were lower than those of the control group.

\subsection{Conclusion}

Through the analysis of the data, it can be concluded that there are strong pirsson correlations among the emergence rate, 100-seed weight and seed volume, which can be used as the basis of seed selection in the future. The differences of emergence rate, 100 -seed weight and seed volume under different treatment time and treatment concentration showed that there might be interaction effect between treatment time and treatment concentration of EMS solution. The difference of plant height and tiller number between control group and treatment group indicated that EMS inhibited seed activity. The results showed that the longer the treatment time and the higher the concentration, the greater the effect on the agronomic characters of Cyperus esculentus L.. The A3 treatment 
group (concentration $0.9 \%$ and treatment time $6 \mathrm{~h}$ ) had the best comprehensive performance in seedling rate, tiller number, plant height and yield. This experiment is the analysis of the agronomic characters of the M0 generation. It is also necessary to analyze the offspring, select the excellent individuals and establish the rich variation pool.

\section{References}

1. Huang Minghua, Wang Xuehua, Pang Zhenyu. Research Status and prospects of Cyperus esculentus L Crop Research 273, 293-295,301 (2013)

2. Zhang Bin, Liu Jing, Fan Zhongxue, Guo Fengdan, Hou Lei, Ma Deyuan, Wayne Wang. Introduction experiment of Cyperus esculentus L.and research progress of green and efficient cultivation techniques J. Shandong Agricultural Sciences, 5103, 147-150 (2019)

3. Han Lijuan, Li Feng. Characteristics and cultivation of Cyperus esculentus L. Special Economic Animals and plants (2003)

4. Yang Zhen LE. Characteristics and research progress of Cyperus esculentus L.J. Northern Horticulture, 192-201. (2017)

5. Cui Xia, Liang Yan, Li Cui, Qin Lei, Li Yunzhou. Chemical mutagenesis and its application in vegetable breeding J. Proceedings of the Northwest A\&F University, 4103, 205-212. (2013)

6. Wang Yun, Zhang Xingguo, Li Hemin, Song Jiajing, Cui Dangqun, Yin Dongmei. Agronomic traits and quality analysis of the mutagenic progenies of peanut EMS J. Journal of Plant genetic resources, 1604, 914-919 + 926. (2015)

7. Feng Wuyi, Wang Nan, Li Shuo, Xiang Fengning. Seed quality identification of soybean EMS MUTANT J. Proceedings of the Qilu University of Technology, 3002, 22-26. (2016)

8. Ding Yanqing, Gao Xu, Cheng Bin, Zhang Riyi. Effect of Ems mutagenesis on the seed of Wheat Variety Guixie 3 J. Agro-technical services, 3503, 46-47. (2018)

9. Cheng Jiaowen, Zhong Jian, Cheng Yurou, Hu Fang, Cui Junjie, Hu Kailin. MUTAGENIC condition analysis of MOMORDICA CHARANTIA SEEDS EMS J. Chinese vegetables, 201901, 26-31.

10. Yongsig Kim, Karen S. Schumaker, Jian-kang Zhu. It's called Arabidopsis Protocol. (2006)

11. Zhang Liwei, Niu Tengfei, Zhang Wenhui, etc. . Screening of soybean mutant "Jihuang 13" and establishment of Mutant Library J. Soybean Science, 321, 33-37(2013)

12. Chiu W H, Chandler J, Cnops G, et Al. . Mutations in the tornado-NAD02 Gene Affect Cellular decision in the peripheral zone of the shoot apical Meristem of Aroopsis 
Thaliana J. Plant Molecular Biology, 636, 731-744. (2007)

13. Kurarthy V, Sood S, Dhaliwal H S, et Al. . Identification and mapping of a tiller inhibition gene TIN-3 IN WHEAT J. Theologii-cal and Applied of Genetics, 1142, 285-294 (2007)

14. Xu Yanhua, Fung Permadi, Dong Mideast, etc. . Construction and preliminary analysis of Mutant Pool of common wheat Yunong 201 induced by EMS J. Journal of Wheat Crops, 304, 625-629 (2010)

15. Krishnan A, Guiderdoni E, An G, et al. Mutant resources in rice forthefunctionalgenomicsofthegrasses, PlantPhysiology, 149, 165-170 (2009)

16. Liu Wei, Yang Feng, Ren Xudong, Zhang Lu, Wu Nan, Tang Fan, Long Yanxi, Ma Jian, Wang Ningning. Advances in the effect of EMS on seed Germination J. Molecular plant breeding, 1511, 4585-4589 (2017)

17. Bai Hongyan, Yang Wei, Wu Bo. Studies on mutagenic treatment of sorghum seed EMS J. China seed industry, 03, 57-59 (2018) 